\title{
Marker Identification and Classification of Cancer Types Using Gene Expression Data and SIMCA
}

\author{
S. Bicciato, A. Luchini, C. Di Bello \\ Department of Chemical Process Engineering, University of Padova, Italy
}

\begin{abstract}
Summary
Objectives: High-throughput technologies are radically boosting the understanding of living systems, thus creating enormous opportunities to elucidate the biological processes of cells in different physiological states. In particular, the application of DNA microarrays to monitor expression profiles from tumor cells is improving cancer analysis to levels that classical methods have been unable to reach. However, molecular diagnostics based on expression profiling requires addressing computational issues as the overwhelming number of variables and the complex, multiclass nature of tumor samples. Thus, the objective of the present research has been the development of a computational procedure for feature extraction and classification of gene expression data.

Methods: The Soft Independent Modeling of Class Analogy (SIMCA) approach has been implemented in a data mining scheme, which allows the identification of those genes that are most likely to confer robust and accurate classification of samples from multiple tumor types.

Results: The proposed method has been tested on two different microarray data sets, namely Golub's analysis of acute human leukemia [1] and the small round blue cell tumors study presented by Khan et al. [2]. The identified features represent a rational and dimensionally reduced base for understanding the biology of diseases, defining targets of therapeutic intervention, and developing diagnostic tools for classification of pathological states.

Conclusions: The analysis of the SIMCA model residuals allows the identification of specific phenotype markers. At the same time, the class analogy approach provides the assignment to multiple classes, such as different pathological conditions or tissue samples, for previously unseen instances.
\end{abstract}

\section{Keywords}

Gene expression data, SIMCA, PCA, feature extraction, classification

Methods Inf Med 2004; 43: 4-8

\section{Introduction}

High-throughput technologies are radically boosting the understanding of living systems, thus creating enormous opportunities to identify target genes and pathways for drug development and to elucidate networks of genomic regulation by the comparison of the phenotype of cells in different physiological states. At present, in the so-called post-genomic era, the accent in biological research is shifting from data acquisition to data analysis and interpretation. Indeed, the increasing pace of genomic data accumulation poses the challenge to develop analysis procedures able to generate new knowledge and upgrade the information content of these databases.

Several different methods have been proposed to analyze large amounts of expression profiling data and identify set of genes that can serve as diagnostic platforms. Among all, the most widely used technique is hierarchical agglomerative clustering. As reported in many publications, clustering techniques have been applied to identify groups of genes sharing similar expression profiles and the results obtained so far are extremely valuable. Clustering techniques have been demonstrated to be useful tools in grouping functional related families of genes. However, clustering methodologies represent an example of unsupervised analysis that is not appropriate for the incorporation of prior knowledge about the observations, as for example sample labels (i.e., normal or tumor tissue), in the partitioning and grouping procedure. As such, cluster analysis may not be a good framework for diagnosis or classification of diseases nor to pinpoint specific features marking a phenotype.

Several machine learning methods have been applied to classify pathologies and tissue samples on the basis of their expression profiles $[1,2]$. This task presents a major challenge due to the overwhelming number of variables (genes), the majority of which is not relevant to the description of the problem and could potentially degrade the performance of the classification scheme by masking the contribution of the relevant features. Thus, together with the development of classification schemes, it is of paramount importance to identify those genes that are most likely to confer high classification accuracy (gene selection). Indeed, these key informative features represent a base of reduced cardinality for subsequent experimental investigation aimed at determining their role, if any, in the generation and progression of the analyzed phenotype.

The purpose of this work is to present a procedure for detecting patterns of expression correlated to peculiar phenotypes through a supervised analysis of labeled samples in the context of multiple tumor types. Specifically, it presents results from a computational framework based on principal component analysis (PCA) and on the Soft Independent Modeling of Class Analogy (3). This approach simultaneously allows identifying specific markers of phenotypes and predicting the class label of a set of previously unseen instances.

The properties of principal component analysis are used to implement a modeling scheme called SIMCA, which has been previously applied to solve many pattern recognition and classification problems. In a multi-class problem, SIMCA works considering each class separately. For each class, a principal component analysis is performed leading to a different PCA model for each class (thus called disjoint class models). Since the models are disjoint, the system describing one class does not depend on 
SIMCA Analysis of Gene Expression Data

that of another category. When classifications of unknown samples are attempted, a comparison is made between the unknown's data and each class model. The model that best fits the unknown, if any, represents the class assigned to that sample. Even if reliable classification of previously unseen instances is the ultimate goal of this approach, SIMCA can also be used for the fundamental issue of feature selection. Indeed, examining the variance structure explained by each model, it is possible to distinguish among the most important variables characterizing each single class and identify specific genes most highly correlated with the tumor type distinctions.

The SIMCA modeling approach has been applied to the analysis of two gene expression databases, namely the data set from Golub's work on leukemia classification [1] and the study presented by Khan et al. [2] on small round blue cell tumors.

\section{Materials and Methods}

\subsection{Gene Expression Data from Tumor Samples}

Two gene expression data sets have been used to illustrate the gene selection and classification method. The leukemia study provides measurements for 3930 probes in 72 samples collected from acute leukemia patients. Forty-seven cases were diagnosed as acute lymphoblastic leukemia (ALL) and the other 25 , as acute myeloid leukemia (AML). The ALL class was further subdivided in $38 \mathrm{~B}$-lineage and $9 \mathrm{~T}$-lineage ALL samples. RNA prepared from bone marrow and peripheral blood cells was hybridized to high-density oligonucleotide microarrays, produced by Affymetrix (Santa Clara, CA). The second database consists of gene-expression data from cDNA experiments describing four childhood malignancies: neuroblastoma (NB), rhabdomyosarcoma (RMS), non-Hodgkin lymphoma (NHL), and the Ewing family of tumors (EWS). The 63 training samples included both tumor biopsy material (13 EWS and 10 RMS) and cell lines (10 EWS, 10 RMS,
$12 \mathrm{NB}$ and $8 \mathrm{BL}$ ). An independent set of 20 blind test samples has been used for testing the classification capabilities of the proposed approach.

\subsection{The SIMCA Method}

Soft Independent Modelling of Class Analogy (SIMCA) uses PCA to extract different characteristics from a set of objects. These characteristics are then used to divide the set into different classes, defined by the user. An object is classified as belonging to the class it resembles the most. The method is discussed in [3-4].

PCA is a statistical data analysis technique that allows reducing the dimensionality of the system while preserving information on variable interactions [4]. PCA transforms the original variables into a set of linear combinations, the principal components (PC), with special properties in terms of variances. Specifically, it deter- mines an optimal linear transformation $\mathbf{y}=$ $\mathbf{W} \mathbf{x}$ of an $n$-dimensional data vector $\mathbf{x}$ into another $m$-dimensional $(m \leq n)$ transformed vector $\mathbf{y}$. The $m x n$ fixed linear transformation matrix $\mathbf{W}$ is designed exploring statistical correlations among the variables of the original data matrix and finding reduced compact data representations that retain maximum nonredundant and uncorrelated intrinsic information of the original data. Exploration of the original data set is based on computing and analyzing the data covariance matrix, its eigenvalues and corresponding eigenvectors organized in descending order. Each element of the $m$-dimensional transformed feature vector $\mathbf{y}$ will be linearly independent and in decreasing order according to decreasing information content. This allows a straightforward reduction of the dimensionality by discarding the feature elements with lower information content. Thus, all original $n$-dimensional data patterns can be optimally transformed to data

\section{Table 1 B-ALL top 20 markers}

\begin{tabular}{|l|l|l|}
\hline Accession number & Symbol & Name \\
\hline X82240 & TCL1A & T-cell leukemia/lymphoma 1A \\
M89957 & CD79B & CD79B antigen (immunoglobulin-associated beta) \\
L33930 & CD24 & CD24 antigen (small cell lung carcinoma cluster 4 antigen) \\
L08895 & MEF2C & MADS box transcription enhancer factor 2, polypeptide C \\
Z49194 & POU2AF1 & POU domain, class 2, associating factor 1 \\
U52682 & IRF4 & Interferon regulatory factor 4 \\
X55740 & NT5E & 5' nucleotidase, ecto (CD73) \\
D88270 & VPREB1 & (lambda) DNA for immunoglobin light chain \\
U05259 & CD79A & MB-1 gene \\
U36922 & FKHR & Fork head domain protein (FKHR) mRNA, 3' end \\
U18259 & MHC2TA & MHC class II transactivator \\
K01911 & NPY & Neuropeptide Y \\
U46006 & CSRP2 & Cysteine and glycine-rich protein 2 \\
X99920 & S100A13 & S100 calcium binding protein A13 \\
U10485 & LRMP & Lymphoid-restricted membrane protein \\
M38690 & CD9 & CD9 antigen (p24) \\
M84371 & CD19 & CD19 gene \\
U49020 & MEF2A & Myocyte-specific enhancer factor 2A, C9 form \\
X58529 & IGHM & Immunoglobulin heavy constant mu \\
X74301 & & MHC class II transactivator \\
\hline
\end{tabular}


patterns in a feature space with lower dimensionality. The algorithm chosen for this work is based on singular value decomposition (SVD) and, since several texts cover the calculation of the PC's in details (e.g., [4]), theoretical aspects will be omitted.

Once a principal component model is calculated, new object data can be projected onto the PC vector space and the total residuals between the predicted and the original data represent a measure of how well the projected data fit the original model. Comparing, through an F-test, the prediction errors with the residual limits calculated for the data used in the model construction (training set), it is possible to see if the sample belongs to the modeled class or not.

With the SIMCA technique, different classes are modeled individually by a separate principal component model. The number of significant PC's is determined for each class. The residuals are used for the creation of boundaries around each class. The distance or standard deviation $s_{k}^{K}$ of object $k$, described by $m$ variables, to a class $K$, modeled with $p_{K}$ principal components, is given by the sum over the $m$ variables of the distances (or residuals) between object $k$ and the PC model along each variable $j\left(e_{k j}^{K}\right)$.

A fundamental issue that can be addressed using the SIMCA modeling scheme is variable selection, meaning the identification of those peculiar features that better characterize a category. The feature selection procedure comprises three major steps: i) identification of those variables that best describe any given class (i.e. the creation of class-specific lists of genes based on the modeling power of the original variables), ii) scoring and ranking of the variables in each class-related list according to their ability to discriminate the class they model from all the other categories, iii) computation of the minimum number of variables needed to maximize multiclass classification. Specifically, comparing the different values of $e_{k j}^{K}$ (i.e., the variance of

\section{Table 2 T-ALl top 20 markers}

\begin{tabular}{|l|l|l|}
\hline Accession number & Symbol & Name \\
\hline X00437 & TRB & T cell receptor beta locus \\
M28826 & CD1B & CD1B antigen, b polypeptide \\
X76223 & MAL & MAL gene exon 4 \\
X04145 & CD3G & CD3G antigen, gamma polypeptide (TiT3 complex) \\
X03934 & CD3D & CD3D antigen, delta polypeptide (TiT3 complex) \\
U23852 & LCK & T-lymphocyte specific protein tyrosine kinase p56lck (LCK) \\
X59871 & TCF7 & Transcription factor 7 (T-cell specific, HMG-box) \\
HG4128-HT4398 & & Anion Exchanger 3, Cardiac Isoform \\
U50743 & FXYD2 & FXYD domain-containing ion transport regulator 2 \\
X14975 & CD1E & CD1E antigen, e polypeptide \\
J04132 & CD3Z & CD3Z antigen, zeta polypeptide (TiT3 complex) \\
M26692 & LCK & T-lymphocyte-specific protein tyrosine kinase (LCK) \\
U40271 & PTK7 & PTK7 protein tyrosine kinase 7 \\
U49835 & CHI3L2 & Chitinase 3-like 2 \\
X87241 & FAT & FAT tumor suppressor homolog 1 (Drosophila) \\
L10373 & TM4SF2 & Transmembrane 4 superfamily member 2 \\
M23323 & CD3E & T-cell surface glycoprotein CD3 epsilon-chain \\
U14603 & CD7 & Protein tyrosine phosphatase type IVA, member 2 \\
M37271 & CD7 antigen (p41) \\
X60992 & CD6 antigen \\
\hline
\end{tabular}

variable $j$ of object $k$ in class model $K$ ), it is possible to sort and rank the different descriptors of the system in terms of their ability to describe a specific category while discriminating among the different classes. A class- $K$-variable is defined so that it presents large values of the residuals when class-K-samples are fitted to all categories but the true $\mathrm{K}$ model and, at the same time, the error of the $\mathrm{K}$ model is minimized only by class-K-samples. The entire procedure has been implemented in Matlab.

\section{Results and Discussion}

\subsection{Leukemia Data Set}

Following the experimental setup described in [1], the data has been split into a training set consisting of 38 samples (19 BALL, 8 T-ALL, and 11 AML) and a blind test set of 34 samples (19 B-ALL, 1 T-ALL, and 14 AML). With the aim to first quantify the relative relevance of each transcript in describing the three different subtypes of leukemia, three PCA models are built using the three groups of training samples after autoscaling the expression levels. A total of 4,4 , and 2 principal components accounting for the 71.9, 73.2, and $88.5 \%$ of the overall variance, respectively, describe the SIMCA models. The number of principal components has been determined using a leaveone-out cross-validation procedure (details described in [4]). Each of the 7129 variables have been assigned to one of the classes analyzing the sum of the residuals produced when a class-K-sample is fitted to all models but the true one, checking, at the same time, the unique minimization of model K residuals for class-K-samples only. The selected genes have been finally sorted combining their modeling power with their discriminating power, among the different classes. This procedure identifies $615 \mathrm{~B}$ lineage ALL, 2657 T-lineage ALL, and 658 AML related transcripts. Tables 1, 2, and 3 list the top 20 markers for each of the three subtypes of leukemia. For all of these features, experimental evidences prove or suggest an important role in acute B-cell 
lymphoblastic, T-cell lymphoblastic, and myeloid leukemias, respectively [1].

The accuracy in the classification of the blind test set improves when using a subset of the modeling features, as compared to all the transcripts or to any random selection of them. Indeed, the classification accuracy arises from $53 \%$ of correct predictions when using all the expression profiles to $82 \%$ of correct predictions using the top $20 \div 40$ markers identified by the SIMCA approach. The definition of unified criteria for the selection of an optimal (or near optimal) subset of markers is under development.

\subsection{Small, Round Blue Cell Tumor Data Set}

The 63 training samples included both tumor biopsy material and cell lines for a total of 4 different categories (EWS, RMS, $\mathrm{NB}$, and $\mathrm{BL}$ ). An independent set of 20 blind test samples has been used for testing the classification capabilities of the proposed approach. Similarly to case study 1 , SIMCA modeling scheme identifies 600 , 496, 512, and 700 genes related to EWS, RMS, NB, and BL respectively. For sake of space, Table 4 lists only the top 15 markers of each category. Most of these transcripts are included in Khan's list of top ranking genes [2]. The classification accuracy improved from $10 \%$ of correct calls, obtained designing the classifier with all the 2308 genes, to $95 \%$ when using only the top $10 \div 15$ markers of each category.

\section{Conclusions}

DNA microarrays are radically boosting the understanding of living systems, thus creating enormous opportunities to elucidate the biological processes of cells in different physiological states. In particular, the application of high-throughput technologies is improving cancer analysis to levels that classical methods have been unable to reach. However, cancer analysis and classification on the basis of microarray data
Table 3 AML top 20 markers

\begin{tabular}{|l|l|l|}
\hline Accession number & Symbol & Name \\
\hline M84526 & DF & D component of complement (adipsin) \\
Y00339 & CA2 & Carbonic anhydrase II \\
M27891 & CST3 & Cystatin C (amyloid angiopathy and cerebral hemorrhage) \\
M96326 & AZU & Azurocidin gene \\
M20203 & ELA2 & Neutrophil elastase gene, exon 5 \\
M31551 & PAI2 & Plasminogen activator inhibitor, type II (arginine-serpin) \\
M31166 & PTX3 & Pentaxin-related gene, rapidly induced by IL-1 beta \\
M27783 & ELA2 & Elastase 2, neutrophil \\
X95735 & ZYX & Zyxin \\
U05572 & MAN2B1 & Mannosidase, alpha, class 2B, member 1 \\
M30703 & AR & Amphiregulin (AR) gene \\
M57731 & GRO2 & GRO2 oncogene \\
L08177 & EBI2 & Epstein-Barr virus induced gene 2 \\
M21119 & LYZ & Lysozyme (renal amyloidosis) \\
X97748 & PTX3 & PTX3 gene promotor region \\
J04990 & CTSG & Cathepsin G \\
M23197 & CD33 & CD33 antigen (gp67) \\
U46751 & SQSTM1 & Sequestosome 1 \\
M28130 & IL8 & Interleukin 8 gene \\
L05424 & CD44 & CD44 gene (cell surface glycoprotein CD44) \\
\hline
\end{tabular}

Table 4 Small, round blue cell tumors top 15 markers

\begin{tabular}{|c|c|c|c|c|c|c|c|}
\hline \multicolumn{2}{|c|}{ EWS } & \multicolumn{2}{|c|}{ RMS } & \multicolumn{2}{|c|}{ NB } & \multicolumn{2}{|c|}{ BL } \\
\hline Clone ID & Symbol & Clone ID & Symbol & Clone ID & Symbol & Clone ID & Symbol \\
\hline 866702 & PTPN13 & 244618 & & 44563 & GAP43 & 183337 & HLA-DMA \\
\hline 770394 & FCGRT & 298062 & TNNT2 & 135688 & GATA2 & 767183 & HCLS1 \\
\hline 377461 & CAV1 & 784224 & FGFR4 & 395708 & DPYSL4 & 740604 & ISG20 \\
\hline 43733 & GYG2 & 461425 & MYL4 & 812105 & AF1Q & 769657 & PPP1R2 \\
\hline 357031 & TNFAIP6 & 770059 & HSPG2 & 383188 & RCV1 & 241412 & ELF1 \\
\hline 814260 & FVT1 & 25725 & FDFT1 & 629896 & MAP1B & 200814 & MME \\
\hline 1473131 & TLE2 & 789253 & PSEN2 & 308231 & MYO1B & 80109 & HLA-DQA1 \\
\hline 1435862 & MIC2 & 769716 & NF2 & 377048 & MYO1B & 236282 & WAS \\
\hline 52076 & OLFM1 & 796258 & SGCA & 220096 & CNGB1 & 624360 & PSMB8 \\
\hline 80338 & SELENBP1 & 1409509 & TNNT1 & 743229 & NEF3 & 530185 & CD83 \\
\hline 1471841 & ATP1A1 & 245330 & IGF2 & 784257 & KIF3C & 609663 & PRKAR2B \\
\hline 365826 & GAS1 & 813841 & PLAT & 878280 & CRMP1 & 47475 & PIR121 \\
\hline 308497 & HT036 & 898219 & MEST & 325182 & $\mathrm{CDH} 2$ & 840942 & HLA-DPB 1 \\
\hline 364934 & DAPK1 & 246035 & -- & 823886 & -- & 68977 & PSMB10 \\
\hline 767345 & -- & 755750 & NME2 & 842918 & FARP1 & 814526 & RNPC1 \\
\hline
\end{tabular}


poses the challenge to develop computational procedures able to address specific issues, such as modeling multiple, heterogeneous populations and reducing the overwhelming number of variables (genes).

The present work addresses the implementation of a multivariate procedure that allows marker identification by extracting transcriptional features of physiological state and sample diagnosis by classifying tumor specimens through the supervised analysis/comparison of expression profiles from multiple tumor types. The gene selection and sample classification scheme is based on Soft Independent Modeling of Class Analogy (SIMCA) and relies on the calibration of a principal component model for each class present in the analyzed data set. In the context of gene expression analysis, the original SIMCA design has been adapted to solve the critical issue of feature selection. In particular, specific subsets of genes most highly correlated with several tumor categories have been identified ex- amining the variance structure explained by each model and evaluating the performance of the classification scheme. SIMCA procedure addresses the multiclass analysis directly with no need to design and combine binary classifiers or preliminary reduce the feature space.

Proof of concept has been given through the analysis of two gene expression databases, namely the data set from a work on leukemia subtypes and a study on small round blue cell tumors. The method has been able to identify groups of genes that could represent bases for subsequent experimental investigations. Moreover, the classification procedure has been able to distinguish with accuracy and robustness between multiple tumor subtypes.

\section{References}

1. Golub TR, Slonim DK, Tamayo P, Huard C, Gaasenbeek M, Mesirov JP, Coller H, Loh M, Downing JR, Caligiuri MA, Bloomfield CD, Lander ES. Molecular Classification of Can- cer: Class Discovery and Class Prediction by Gene Expression Monitoring. Science 1999; 286 (5439): 531-7.

2. Khan J, Wei JS, Ringner M, Saal LH, Ladanyi M, Westermann F, Berthold F, Schwab M, Antonescu CR, Peterson C, Meltzer PS. Classification and diagnostic prediction of cancers using gene expression profiling and artificial neural networks. Nat Med 2001; 7 (6): 673-9.

3. Wold S, Sjostrom M. SIMCA: a method for analyzing chemical data in terms of similarity and analogy. Kowalski BR, editor. Chemometrics: Theory and Application. Washington: ACS; 1977.

4. Jolliffe I. Principal Components Analysis. Springer-Verlag; 1986.

\section{Correspondence to:}

Dr. Silvio Bicciato

Department of Chemical Process Engineering

University of Padova

Via Marzolo, 9

35131 Padova, Italy

E-mail: silvio.bicciato@unipd.it 